\title{
STRONG CONVERGENCE OF SELECTIONS IMPLIED BY WEAK
}

\author{
TADEUSz RzEZUCHOWSKI
}

\begin{abstract}
In some situations weak convergence in $\mathcal{L}_{1}$, implies strong convergence. Let $P, L: T \rightarrow$ $\mathrm{Co}\left(\boldsymbol{R}^{d}\right)$ be measurable multifunctions $\left(\mathrm{Co}\left(\boldsymbol{R}^{d}\right)\right.$ being the set of closed, convex subsets of $\left.\mathbf{R}^{d}\right)$, the values $L(t)$ affine sets and $W(t)=P(t) \cap L(t)$ extremal faces of $P(t)$. Let $p_{k}$ be integrable selections of $P, \bar{p}_{k}(t)$ the projection of $p_{k}(t)$ on $L(t)$ and $\bar{p}_{k}(t)$ on $W(t)$. We prove that if $p_{k}-\bar{p}_{k}$ converges weakly to zero then $p_{k}-\bar{p}_{k}$ converges to zero in measure. We give also some extensions of this theorem. As applications to differential inclusions we investigate convergence of derivatives of convergent sequences of solutions and we describe solutions which are in some sense isolated. Finally we discuss what can be said about control functions $u$ when the corresponding trajectories of $\dot{x}=f(t, x, u)$ are convergent to some trajectory.
\end{abstract}

\section{Preliminary remarks on measurable multifunctions}

Multifunctions are maps whose values are sets. There exist exhaustive references to the fundamental properties of multifunctions - see $[\mathbf{1}, \mathbf{3}]$. We shall discuss here, as briefly as possible, those that are essential to the results given in the sequel.

Throughout the paper, $T$ will be a space endowed with a $\sigma$-field $\mathcal{L}$ and a $\sigma$-finite, complete measure $m$.

For a metric space $Y$ by $C l(Y)$ we mean the collection of its closed subsets; $C o\left(\mathbf{R}^{d}\right)$ is the subfamily of $C l\left(\mathbf{R}^{d}\right)$ composed of convex sets.

For $A, B \subseteq Y$ we put:

$$
\begin{gathered}
e(A, B)=\sup \{\operatorname{dist}(a, B) a \in A\} \\
H(A, B)=\max \{e(A, B), e(B, A)\}
\end{gathered}
$$

$H$ is called Hausdorff metric when applied to $A, B$ closed.

A multifunction $P: T \rightarrow C l(Y)$ is said to be measurable if for every open $U \subset Y$ the set $\{t \in T ; P(t) \cap U \neq \emptyset\}$ is measurable. A fundamental property of measurable multifunctions, for $Y$ separable and complete, is the existence of a measurable selection, that means a measurable function $f(t) \in P(t)$ - known as the Theorem of Kuratowski and Ryll-Nardzewski - and its stronger version, due to Castaing, stating that there is

\section{Received 28 April 1988}

The author is indebted to Professor C. Castaing for providing him with the references [2] and [11] closely related to the subject of this paper.

Copyright Clearance Centre, Inc. Serial-fee code: 0004-9729/89 \$A2.00+0.00. 
a sequence of measurable selections $f_{n}$ such that the sets $\left\{f_{n}(t) ; n \in N\right\}$ are dense in $P(t)$ a.e. in $T$. All this can be found in [3].

If $Y$ is separable and complete then there is a very useful characterisation of measurability of a multifunction through the measurability of its graph $G(P)=\{(t, y) ; y \in$ $P(t)\}$ in the product $\sigma$-field $\mathcal{L} \times \mathcal{B}$, where $\mathcal{B}$ is the Borel $\sigma$-field in $Y$. Applying it one can prove easily many useful properties, for example the measurability of denumerable intersections of measurable multifunctions. Moreover, if $G(P)$ is measurable and if the values $P(t)$ are non-empty then $P$ has a measurable selection even if the $P(t)$ are not closed. All this is given in detail in [3, chapter 3.4].

If the domain $X$ of a multifunction $P: X \rightarrow C l(Y)$ is a metric space then we can speak about other kinds of regularity of $P$. It is upper semicontinuous (usc) if the set $\{x \in X ; P(x) \cap C \neq \emptyset\}$ is closed for every closed $c \subseteq Y$. For compact-valued multifunctions upper semicontinuity is equivalent to the implication $x_{n} \rightarrow x \rightarrow e\left(P\left(x_{n}\right), P(x)\right) \rightarrow 0$, for every $x$. Continuity is understood with respect to the Hausdorff metric in the range.

Especially important are convex-valued multifunctions. Before discussing their properties we recall some facts about convex sets - a reference for these is [8]. The scalar product of $x, y \in R^{d}$ will be denoted by $\langle x, y\rangle$. If $C \in C o\left(\mathbf{R}^{d}\right), y, z \in \mathbf{R}^{d}$ then

$$
\begin{gathered}
\operatorname{supp}(C, y)=\sup \{\langle x, y\rangle ; x \in C\}, \\
S(C, y)=\left\{x \in R^{d} ;\langle x, y\rangle=\operatorname{supp}(C, y)\right\}, \\
N(C, z)=\left\{u \in \mathbf{R}^{d} ; \forall x \in C\langle x-z, u\rangle \leqslant 0\right\} .
\end{gathered}
$$

$N(C, z)$ is the normal cone to $C$ at the point $z$. It is closed convex and for $z \in C, y \in N(C, z)$ we have $z \in C \cap S(C, y)$.

A face of $C \in C o\left(R^{d}\right)$ is any subset $D$ such that if $a, b \in C, a \neq b$ and $\lambda a+$ $(1-\lambda) b \in D$ for some $\lambda \in(0,1)$ then $a, b \in D$. Faces are closed and convex. If $D$ is a $k$-dimensional affine set $L$ in $\mathbf{R}^{d}$ for which $D C \cap L$.

The notion of lexicographical order in $\mathbf{R}^{d}$ is strictly connected with the above. Recall that the lexicographical order induced by the set of linearly independent vectors $\left\{e_{1}, \ldots, e_{k}\right\}=E$ is defined by: $x \leqslant E y$ if and ony if the first nonzero product $\left\langle e_{j}, y-x\right\rangle$ is positive. This relation is transitive and for every $x, y \in \mathbf{R}^{d}$ either $x \leqslant_{E} y$ or $y \leqslant_{E} x$. But $x \leqslant_{E} y$ and $y \leqslant_{E} x$ imply $x=y$ only if $k=d$.

A point $x \in C \subseteq \mathbf{R}^{d}$ is said to be maximal in $C$ if $y \leqslant_{E} x$ for all $y \in C$. If $C \in C o\left(\mathbf{R}^{d}\right)$ then the set of all maximal points in $C$ is a face of dimension less than or equal to $d-k$.

Conversely, if $D$ is a $(d-k)$-dimensional face of a convex, closed set $C$ then there 
exists a set $\left\{e_{1}, \ldots, e_{k}\right\}=E$ of orthonormal vectors such that $D$ is the set maximal points in $C$ with respect to the order $\leqslant E$.

We shall often put a dot in the place of a variable for example $f(t, \cdot)$ is the function $g(x)=f(t, x)$, where $t$ is fixed.

LEMMA 1.1. If $P: T \rightarrow C o\left(\mathbf{R}^{d}\right)$ and $p: T \rightarrow \mathbf{R}^{d}$ are measurable then $N(P(\cdot), p(\cdot))$ is a measurable multifunction.

Proof: Let $q_{n}$ be measurable selections of $P$ with $\left\{q_{n}(t) n \in N\right\}$ dense in $P(t)$ a.e. in $T$. We put

$$
M_{n}(t)=\left\{y \in \mathbf{R}^{d} ;\left\langle y, q_{n}(t)-p(t)\right\rangle \leqslant 0\right\} .
$$

The multifunctions $M_{n}$ are measurable and $N(P(t), p(t))=\cap\left\{M_{n}(t) n \in \mathrm{N}\right\}$ which proves the assertion.

Now let a measurable multifunction $P: T \rightarrow C o\left(R^{d}\right)$ be fixed. We shall discuss some properties of multifunctions whose values are faces of $P(t)$.

Let $e_{1}, \ldots, e_{d-k}: T \rightarrow \mathbf{R}^{d}$, where $0 \leqslant k<d$, be measurable functions with the values $\left\{e_{1}(t), \ldots, e_{d-k}(t)\right\}=E(t)$ linearly independent a.e. in $T$. We denote by $W(t)$ the set of all maximal points in $P(t)$ with respect to the order $\leqslant_{E(t)}$.

LEMMA 1.2. The $W(t)$ are faces of $P(t)$ of dimension less then or equal to $k$ and the multifunction $W$ is measurable.

Proof: We put $W_{0}(t)=P(t)$ and define by recurrence

$$
W_{j}(t)=W_{j-1}(t) \cap S\left(W_{j-1}(t), e_{j}(t)\right) .
$$

Then $W(t)=W_{d-k}(t)$ and thus $W$ is measurable.

Conversely, the following lemma holds.

LEMMA 1.3. If $W: T \rightarrow C o\left(\mathrm{~A}^{d}\right)$ is measurable and its values are faces of $P(t)$ of dimension $k$, where $0 \leqslant k \leqslant d$, then there exist measurable functions $e_{1}, \ldots, e_{d-k}: T \rightarrow \mathbf{R}^{d}$ with values $\left\{e_{1}(t), \ldots, e_{d-k}(t)\right\}=E(t)$ orthonormal a.e. in $T$ and such that $W(t)$ are the sets of maximal points in $P(t)$ with respect to $\leqslant E(t)$.

Proof: In the case $k=d$ we have $E(t)=\emptyset$ and the theorem is trivial. $W(t)=$ $P(t)$ and all points in $P(t)$ are maximal.

We fix $k<d$. Let $q$ be some measurable selection of $W$. We put $W_{0}(t)=P(t)$ and $L_{0}(t)=\mathbf{R}^{d}$. We show now how to find $W_{j}, L_{j}$ and $e_{j}$ if $W_{j-1}, L_{j-1}$ are known - the $W_{j}$ will be faces of $P(t)$ lying in affine sets $L_{j}(t)$, and the $e_{j}(t)$ will be vectors normal to $W_{j-1}(t)$ at $q(t)$. As long as $j<d-k q(t)$ belongs to the boundary of $W_{j-1}(t)$ with respect to the euclidean topology in $L_{j-1}(t)$, so the intersections

$$
N\left(W_{j-1}(t), q(t)\right) \cap S_{d-1} \cap\left(L_{j-1}(t)-q(t)\right)
$$


are non-empty and define a measurable, closed-value multifunction ( $S_{d-1}$ is the unit sphere in $\left.R^{d}\right)$. We can pick up its measurable selection $e_{j}$ and put $L_{j}(t)=L_{j-1}(t) \cap$ $S\left(W_{j-1}(t), e_{j}(t)\right)$. The functions $e_{1}, \ldots, e_{d-k}$ satisfy the desired properties.

Let us remark that the multifunction $L(t)=L_{d-k}(t)$ from the above proof is measurable, its values are $k$-dimensional affine subsets of $\mathbf{R}^{d}$ and $W(t)=P(t) \cap L(t)$. Our assumption on the dimension of $W(t)$ implies that $L$ is the unique multifunction having these properties.

Lemma 1.3 can be slightly modified to yield:

Lemma 1.4. If $L: T \rightarrow C o\left(R^{d}\right)$ is a measurable multifunction whose values are affine sets of dimension $k$ and $W(t)=P(t) \cap L(t)$ are extremal faces of $P(t)$ then there exist measurable functions $e_{1}, \ldots, e_{d-k}: T \rightarrow R^{d}$ with values $\left\{e_{1}(t), \ldots, e_{d-k}(t)\right\}=$ $E(t)$ orthonormal, orthogonal to the linear subspace parallel to $L(t)$ and such that $W(t)$ are the sets of maximal points in $P(t)$ with respect to $\leqslant E(t)$.

(Note that the dimension of $W(t)$ may be for some $t$ less than $k$ ).

Now let $p$ be a measurable selection of a measurable multifunction $P: T \rightarrow$ $C o\left(R^{d}\right)$. We shall discuss the properties of the multifunction $W(t)$ whose values are faces of $P(t)$ containing $p(t)$ and of minimal dimension. The following property is useful.

Lемма 1.5. Let $Q, L: \rightarrow C o\left(R^{d}\right)$ be measurable multifunctions, the values $L(t)$ affine sets and $Q(t) \subseteq L(t)$ a.e. in $T$. Then the multifunction $\partial_{L(t)} Q(t)$ is measurable. (Its values are the boundaries of $Q(t)$ with respect to the euclidean topology in $L(t)$.)

Proof: Let $q_{n}$ be measurable selections of $L$ such that the sets $\left\{q_{n}(t) n \in \mathbf{N}\right\}$ are dense in $L(t)$. The functions $v_{n}(t)=\operatorname{dist}\left(q_{n}(t)\right), Q(t)$ are measurable. Putting $B_{n}(t)=B\left(q_{n}(t), v_{n}(t)\right)$ if $v_{n}(t)>0$ and $B_{n}(t)=\emptyset$ otherwise, we can express the boundary in the following way

$$
\partial_{L(t)} Q(t)=\overline{\bigcup_{n \in N}\left(B_{n}(t) \cap Q(t)\right)}
$$

and this implies the required measurability.

On the basis of the above property and using finite induction the following lemma can be proved.

LEMmA 1.6. Let $P: T \rightarrow C o\left(\mathbf{R}^{d}\right)$ be measurable, $p$ its measurable selection and $W(t)$ the face of $P(t)$ containing $p(t)$ of minimal dimension. Then $W$ is measurable and the $U_{k}=\{t \in T ; \operatorname{dim}(W(t))=k\}$ are measurable for $k=0,1, \ldots, d$. 


\section{The MAIN RESUlT}

We shall prove now the basic result of the paper and give some of its direct consequences.

Throughout this section $P, L: T \rightarrow C o\left(\mathbf{R}^{d}\right)$ will be measurable multifunctions; the measure on $T$ is, as before, $\sigma$-finite and complete. We assume that the $L(t)$ are affine sets, and that the $W(t)=P(t) \cap L(t)$ are extremal faces of $P(t)$.

For any function $p: T \rightarrow \mathbf{R}^{d}$, by $\tilde{p}(t)$ we shall mean the projection of $p(t)$ on $L(t)$ and by $\bar{p}(t)$ the projection on $W(t)$. If $p$ is measurable then both $\tilde{p}$ and $\bar{p}$ are also measurable.

Speaking of weak convergence, compactness, etcetera we shall always mean the weak topology in $\mathcal{L}_{1}\left(T, \mathbf{R}^{d}\right)$.

We shall consider a sequence $p_{n}$ of integrable selections of $P$. The following theorem will be the basis for all other results in this paper.

THeOREM 2.1. Assume that $W$ has an integrable selection and that the sequence of selections $p_{n}$ of $P$ is weakly precompact. Then the weak convergence to zero of $p_{n}-\tilde{p}_{n}$ implies convergence in measure to zero of $p_{n}-\bar{p}_{n}$.

Proof: The proof can be reduced to the case when $\operatorname{dim}(L(t))$ does not depend on $t$, since $T$ can be divided into measurable sets $\{t \in T ; \operatorname{dim}(L(t))=j\}, j=0,1, \ldots, d$. So, let us suppose that $\operatorname{dim}(L(t))$ is constant and equal to $k$.

Let the functions $e_{1}, \ldots, e_{d-k}: T \rightarrow \mathrm{P}^{d}$ be as in Lemma 1.4.

We can write $p_{n}-\bar{p}_{n}=\left(p_{n}-\tilde{p}_{n}\right)+\left(\tilde{p}_{n}-\bar{p}_{n}\right)$. We shall prove first that $p_{n}-\bar{p}_{n} \rightarrow 0$ in measure and then the same for $\tilde{p}_{n}-\bar{p}_{n}$.

We put $f_{n, j}(t)=\left\langle e_{j}(t), p_{n}(t)-\bar{p}_{n}(t)\right\rangle$. Then

$$
p_{n}(t)-\tilde{p}_{n}(t)=\sum_{j=1}^{d-k} f_{n, j}(t) e_{j}(t)
$$

We shall prove first by induction on $j$ that the sequences $f_{n, j}$ tend to zero in measure, as $n \rightarrow \infty$.

The functions $f_{n, 1}$ are non-positive so for every $K \subset T$ of finite measure the weak convergence to zero of their restrictions to $K$ implies convergence in measure to zero on $K$. In view of Lemma 2.3 , cited at the end of this section, for any fixed $\alpha, \varepsilon>0$ there exists a set $K \subseteq T$ of finite measure such that

$$
\int_{T \backslash K}\left|f_{n, 1}\right| d m<\frac{\alpha \varepsilon}{2}
$$

for all $n \in \mathrm{N}$, so $m\left(\left\{t \in T \backslash K ;\left|f_{n, 1}(t)\right|>\alpha\right\}\right)<\varepsilon / 2$. From this and the convergence in measure on $K$ we get $m\left(\left\{t \in T\left|f_{n, 1}(t)\right|>\alpha\right\}\right)<\varepsilon$ for $n$ sufficiently great. 
Now, let us suppose that the sequences $f_{n, 1}, \ldots, f_{n, j-1}$ converge in measure to zero as $n \rightarrow \infty$, where $1 \leqslant j<d-k$. We prove first that $f_{n . j} \rightarrow 0$ in measure for any fixed $K \subseteq T$ of finite measure

Let us fix $\alpha, \varepsilon>0$. In view of the boundedness of $p_{n}$ in the norm of $\mathcal{L}_{1}$, there is a closed ball $B$ in $R^{d}$, centred at 0 , such that putting $B_{n}=\left\{t \in K ; p_{n}(t) \in B\right\}$ we have $m\left(K \backslash B_{n}\right)<\varepsilon / 3$ for all $n$.

We define an auxiliary function

$$
\begin{aligned}
w(t, \delta) & =\max \left\{\left\langle e_{j}(t), y-\operatorname{proj}_{W(t)} y\right\rangle ; y \in P(t) \cap B,\right. \\
& \left.\left\langle e_{i}(t), y-\operatorname{proj}_{W(t)} y\right\rangle \geqslant-\delta, \text { for } i=1, \ldots, j-1\right\},
\end{aligned}
$$

where $t \in K, \delta>0$. For every fixed $\delta$ the function $w(\cdot, \delta)$ is measurable and for every fixed $t \in K, \lim _{\delta \rightarrow 0+} w(t, \delta)=0$. Using Egorov's Theorem we can fix $\delta_{0}>0$ and a measurable set $Z \subseteq K$ such that $m(K \backslash Z)<\varepsilon / 3$ and $w(t, \delta) \leqslant \alpha$ for $t \in Z$ and $0<\delta \leqslant \delta_{0}$.

Now let

$$
D_{n}=\bigcup_{i=1}^{j-1}\left\{t \in K f_{n, 1}(t) \geqslant-\delta_{0}\right\} .
$$

The induction hypothesis implies that $m\left(K \backslash D_{n}\right)<\varepsilon / 3$ for $n$ greater than some $n_{0}$.

Let us put $E_{n}=B_{n} \cap Z \cap D_{n}$. For $n \geqslant n_{0}$ we have $m\left(K \backslash E_{n}\right)<\varepsilon$ and, if $t \in E_{n}$, then the inequality $f_{n, j}(t) \leqslant \alpha$ holds. Thus the positive parts of $f_{n, j}$ converge on $K$ in measure to zero and this implies, together with the weak convergence of $f_{n, j}$, that the $f_{n, j}$ converge to zero in measure.

To prove the convergence in measure on all $T$ we proceed exactly as in the case of $f_{n, 1}$. We find, for fixed $\alpha, \varepsilon>0$, a set $K \subseteq T$ of finite measure such that

$$
\int_{T \backslash K}\left|f_{n, j}\right| d m<\frac{\alpha \varepsilon}{2}
$$

for all $n$ and then use the convergence in measure on $K$.

The proof of the convergence $\bar{p}_{n}-\bar{p}_{n} \rightarrow 0$ in measure is almost exactly the same as the second induction step above - instead of $w$ one has to use the auxiliary function

$$
\begin{gathered}
\bar{w}(t, \delta)=\max \left\{\left|\operatorname{proj}_{L(t)} y-\operatorname{proj}_{W(t)} y\right| ; y \in B \cap P(t),\right. \\
\left.\left|y-\operatorname{proj}_{L(t)} y\right| \geqslant \delta\right\}
\end{gathered}
$$

where $B$ is a suitable ball. (That is the only part of the proof where the existence of an integrable. selection of $W$ is needed.) 
Theorem 2.1 could be formulated without using $L$ and the projection $\tilde{p}$. The assumption would be then the weak convergence of $p_{n}-\bar{p}_{n}$ to zero. To prove it in this case one can divide $T$ onto disjoint, measurable subsets on which the dimension of $W(t)$ is constant. On each one there exists a measurable, affine valued multifunction $L$ with $\operatorname{dim}(L(t))=\operatorname{dim}(W(t)), W(t)=P(t) \cap L(t)$ and Theorem 2.1 can be applied.

The assumption in Theorem 2.1 that $p_{n}$ are selections of $P$ can be weakened to some kind of convergence of $p_{n}$ to $P$.

DEFINITION: A sequence of measurable functions $p_{n}: K \rightarrow \mathrm{R}^{d}$, where $K \subseteq T$, converges on $K$ in measure to a measurable multifunction $P: K \rightarrow C l\left(R^{d}\right)$ if for every $\alpha>0$

$$
\lim _{n \rightarrow \infty} m\left(\left\{t \in K \operatorname{dist}\left(p_{n}(t), P(t)\right) \geqslant \alpha\right\}\right)=0
$$

We can give now the following extension of Theorem 2.1.

CoROLlary 2.2. Let all the assumptions of Theorem 2.1 be satisfied except the condition that the $p_{n}$ are selections of $P$. We suppose instead the convergence of $p_{n}$ to $P$ in measure on every set $K \subset T$ of finite measure. Then the assertion of Theorem 2.1 holds.

PROOF: Let $q_{n}(t)$ be the projection of $p_{n}(t)$ on $P(t)$. The existence of integrable selection of $P$ and the weak precompactness of $\left\{p_{n} ; n \in \mathrm{N}\right\}$ imply that the $q_{n}$ are integrable and the set $\left\{q_{n} ; n \in N\right\}$ is weakly precompact. From the equality $q_{n}-$ $\tilde{q}_{n}=\left(q_{n}-p_{n}\right)+\left(p_{n}-\tilde{p}_{n}\right)+\left(\tilde{p}_{n}-\bar{q}_{n}\right)$ we get, using the assumption of convergence of $p_{n}$ to $P$, the weak convergence of $q_{n}-\tilde{q}_{n}$ to zero. Theorem 2.1 and the equality $p_{n}-\bar{p}_{n}=\left(p_{n}-q_{n}\right)+\left(q_{n}-\bar{q}_{n}\right)+\left(\bar{q}_{n}-\bar{p}_{n}\right)$ imply that $p_{n}-\bar{p}_{n} \rightarrow 0$ in measure.

We cite now a lemma on weak compactness in $\mathcal{L}_{1}$ that was used in this section.

LEMMA 2.3. (Theorem 8.9 in [4]) $A$ set $(f)$ of integrable functions is weakly precompact in $\mathcal{L}_{1}$ if and only if the following conditions are satisfied:

(a) it is norm-bounded on $\mathcal{L}_{1}$;

(b) it is uniformly integrable;

(c) for every $\varepsilon>0$ there is a set $K \subset T$ of finite measure such that

$$
\int_{T \backslash K}|f| d m \leqslant \varepsilon
$$

for all $f$ in this set.

In the assertions of Theorem 2.1 and Corollary 2.2 we need the convergence in measure. However, weak compactness in $\mathcal{L}_{1}$ and convergence in measure of a sequence imply its strong convergence in $\mathcal{L}_{1}$. 
Remarks. Theorem 1 in $[\mathbf{1 1}]$ corresponds to the special case of our Theorem 2.1 when the affine sets $L(t)$ are 0 -dimensional, that means $L(t)=W(t)=\left\{p_{0}(t)\right\}$ and $p_{0}(t)$ are extremal points of $P(t)$. The theorem says then that the weak convergence of $p_{k}$ to $p_{0}$ implies their strong convergence. The proof differs from the one given above and is based, roughly speaking, on the fact that if $c$ is an extremal point of a closed, convex set $C$ then for any $r>0$ it can be strongly separated from the convex hull of $C \backslash B(c, r)$.

In [2] Balder considered the case of functions with values in reflexive and separable Banach spaces. He proves a theorem analogous to that above of Visintin - the convergence of $p_{k}$ that he gets is weaker than strong but stronger than weak. The proof is based on the use of Young measures.

In $[6,7]$ Olech gave results which are close to those discussed above. He proved that if $c$ is an extremal point of the closure of $I=\int_{[0,1]} P(t) d t$ (the integral in the sense of Aumann, $P:[0,1] \rightarrow C l\left(\mathbb{R}^{d}\right)$ measurable), $p, q$ are integrable selections of $P$ and $\left|c-\int_{[0,1]} p(t) d t\right|,\left|c-\int_{[0,1]} q(t) d t\right|$ are small then $\int_{[0,1]}|p(t)-q(t)| d t$ is small. This implies that if $p_{k}, p_{0}$ are integrable selections of $P, \int_{[0,1]} p_{k}(t) d t \rightarrow \int_{[0,1]} p_{0}(t) d t$ and $c=\int_{[0,1]} p_{o}(t) d t$ then $p_{k} \rightarrow p_{0}$ strongly in $\mathcal{L}_{1}$. We note that instead of weak convergence it suffices to assume here this convergence of the integrals over all the space. The cost of this is the assumption of extremality of $c$ in $I$. Thus $c$ is maximal in $I$ with respect to some total lexicographical order and $p_{0}(t)$ must be maximal in $P(t)$ with respect to the same, constant order. In our case the order may change with $t$. Olech gave two proofs of his theorem. One is based on the use of lexicographical order [6] and the other [7] on the property of extremal points which we mentioned in relation with the proof of Visintin.

\section{APPLICATIONS TO DIFFERENTIAL INCLUSION}

We shall give two applications. Firstly we shall treat convergent sequences of solutions of a differential inclusion and see what can be said about the convergence of their derivatives. Then we shall discuss some special kinds of solutions which are, in a sense, isolated.

We shall consider a multifunction $F: I \times \mathbf{R}^{d} \rightarrow C o\left(R^{d}\right)$, where $I=[0,1]$, and solutions of the differential inclusion

$$
\dot{x} \in F(t, x)
$$

in the sense of Caratéodory (absolutely continuous functions $x: I \rightarrow \mathrm{R}^{d}$ satisfying $\dot{x}(t) \in F(t, x(t))$ a.e. in I).

Also, $F$ will satisfy the following conditions:

(a1) for every $\varepsilon>0$, there is a closed subset $I_{\varepsilon} \subseteq I$ such that $m\left(I \backslash I_{\varepsilon}\right)<\varepsilon$ 
and the restriction of $F$ to $I_{\varepsilon} \times \mathbf{R}^{d}$ is upper semicontinuous;

(b1) there exist integrable functions $g_{1}, g_{2}: I \rightarrow \mathbf{R}^{+}$such that for every $t \in I$, $x \in \mathbf{R}^{d}, y \in F(t, x)$ the inequality $|y| \leqslant g_{1}(t)|x|+g_{2}(t)$ holds.

Condition (a1) is, in some sense, minimal for differential inclusions with convexvalued right-hand side. This is explained in $[5,9]$.

Assumption (b1) implies that if $\left\{x_{\gamma} ; \gamma \in \Gamma\right\}$, where $\Gamma$ is some set, is a family of solutions of (3.1) and the set of initial conditions $\left\{x_{\gamma}(0)\right\}$ is bounded then there exists an integrable function $g: I \rightarrow \mathbf{R}^{+}$such that for every $\gamma \in \Gamma\left|\dot{x}_{\gamma}(t)\right| \leqslant g(t)$ a.e. in $I$.

Now let $x_{0}, x_{n}$, for $n \in \mathrm{N}$, be solutions of (3.1) and $e_{1}, \ldots, e_{d-k}: I \rightarrow \mathbf{R}^{d}$ measurable functions with values $\left\{e_{1}(t), \ldots, e_{d-k}(t)\right\}=E(t)$ orthonormal a.e. in $I$. By (a1) the multifunction $P(t)=F\left(t, x_{0}(t)\right)$ is measurable.

Let $K \subseteq I$ be measurable.

THEOREM 3.1. If the solutions $x_{n}$ converge pointwise to the solution $x_{0}$ and the derivatives $\dot{x}_{0}(t)$ for $t \in K$ are maximal in $F\left(t, x_{0}(t)\right)$ with respect to the lexicographical order induced by $E(t)$ then for every $j=1, \ldots, d-k$ the sequence $\left\langle e_{j}(\cdot), \dot{x}_{n}(\cdot)-\dot{x}_{0}(\cdot)\right\rangle$ converges in measure to zero on $K$.

Proof: For every $r \in I$ we have $x_{n}(t) \rightarrow x_{0}(t)$ as $n \rightarrow \infty$, so $e\left(F\left(t, x_{n}(t)\right), P(t)\right)$ $\rightarrow 0$. This implies that the sequence $\dot{x}_{n}$ converges in measure to the multifunction $P$. The assumption (b1) and the convergence of $x_{n}$ to $x_{0}$ imply the weak convergence of $\dot{x}_{n}-\dot{x}_{0}$ to zero. To end the proof it suffices to apply Corollary 2.2 .

An important special case of Theorem 3.1 is when $k=0$ - this means that $\dot{x}_{0}(t)$ are extremal points of $F\left(t, x_{0}(t)\right)$ a.e. in $I$.

Corollary 3.2. If the solutions $x_{n}$ converge to the solution $x_{0}$ and $\dot{x}_{0}(t) \epsilon$ $\operatorname{Ext}\left(F\left(t, x_{0}(t)\right)\right)$ a.e. in $I$ then $\dot{x}_{n} \rightarrow \dot{x}_{0}$ in measure. (Ext denotes all extremal points of a set.)

Now we hall discuss a situation that may occur when the sets $F\left(t, x_{0}(t)\right)$, along a solution $x_{0}$, are much bigger than the sets $F(t, x)$ for $x$ near to $x_{0}(t)$.

So, let $x_{0}$ be a solution of (3.1) and consider the following condition:

(c1) almost everywhere in $I$ there exist $r(t)>0$ such that

$$
\dot{x}_{0}(t) \notin \operatorname{conv} \bigcup\left\{F(t, x) ; 0<\left|x-x_{0}(t)\right| \leqslant r(t)\right\}
$$

(conv stands for closed, convex hull.)

THEOREM 3.3. Let the conditions (a1), (b1), (c1) hold. If $x_{n}$ is a sequence of solutions of (3.1) convergent pointwise to the solution $x_{0}$ then

$$
\lim _{n \rightarrow \infty} m\left(\left\{t \in I ; x_{n}(t) \neq x_{0}(t)\right\}\right)=0 .
$$


To prove this theorem we shall need a lemma in which we give to the condition (a3) a form suitable for applying Theorem 3.1 .

LemMA 3.4. Under assumptions (a1), (b1), (c1) there exist measurable functions $q: I \rightarrow R^{+}, u: I \rightarrow R^{d}$ such that $|u(t)|=1$ and

$$
\begin{aligned}
h(t)= & \left\langle\dot{x}_{0}(t), u(t)\right\rangle \\
& -\operatorname{supp}\left(\operatorname{conv} \bigcup\left\{F(t, x) ; 0<\left|x-x_{0}(t)\right| \leqslant q(t)\right\}, u(t)\right)>0
\end{aligned}
$$

a.e. in $I$.

PROOF: Let $q^{*}: I \rightarrow R^{+}$be the measurable function which is greater than or equal to $r$ and less than or equal to any other measurable function which is greater than $r$. We put $q(t)=q^{*}(t) / 2$. The set $C=\{t \in I ; r(t)>q(t)\}$ has the outer Lebesgue measure $m^{*}(C)$ equal to 1 . Moreover, as $m^{*}(\{t \in I ; q(t)>0\})=1$, so $q(t)>0$ a.e. in $I$.

Let us prove that the multifunction

$$
Z(t)=\operatorname{conv} \bigcup\left\{F(t, x) ; 0<\left|x-x_{0}(t)\right| \leqslant q(t)\right\}
$$

is measurable. First we show that for a fixed $\beta>0$ the multifunction

$$
Z_{\beta}(t)=\bigcup\left\{F(t, x) ; \beta \leqslant\left|x-x_{0}(t)\right| \leqslant q(t)\right\}
$$

has $\mathcal{L} \times \mathcal{B}$ measurable graph. To do this let us fix for a moment $n \in \mathrm{N}$ and let $J_{n} \subset I$ be a closed set such that $m\left(I \backslash J_{n}\right) \leqslant 1 / n$, the restriction of $q$ to $J_{n}$ is continuous and of $F$ to $J_{n} \times \mathbf{R}^{d}$ upper semicontinuous with respect to $(t, x)$. The set

$$
A_{n}=\left\{(t, x) \in J_{n} \times \boldsymbol{R}^{d} ; \beta \leqslant\left|x-x_{0}(t)\right| \leqslant q(t)\right\}
$$

is compact so the graph $G\left(\left.F\right|_{A_{n}}\right)$ is also compact in $I \times \mathbf{R}^{d} \times \mathbf{R}^{d}$. Let $\Psi: I \times \mathbf{R}^{d} \times \mathbf{R}^{d} \rightarrow$ $I \times \mathbf{R}^{d}$ be the projection defined by $\Psi(t, x, y)=(t, y) . \quad G\left(Z_{\beta} \mid J_{n}\right)=\Psi\left(G\left(\left.F\right|_{A_{n}}\right)\right)$ so $G\left(Z_{\beta} \mid J_{n}\right)$ is compact. This implies that the graph of the restriction of $Z_{\beta}$ to $\bigcup\left\{J_{n} n \in \mathrm{N}\right\}$ is $\mathcal{L} \times \mathcal{B}$ measurable in $I \times \mathbf{R}^{d}$.

Now consider the multifunction

$$
\tilde{Z}(t)=\bigcup\left\{F(t, x) ; 0<\left|x-x_{0}(t)\right| \leqslant q(t)\right\}
$$

Applying the abvove we deduce that there is a measurable set $J \subset I$ such that $m(I \backslash J)=0$ and the graph $G\left(\left.\bar{Z}\right|_{J}\right)$ is $\mathcal{L} \times \mathcal{B}$ measurable. This implies finally that the multifunction $Z(t)=\operatorname{conv} \tilde{Z}(t)$ is measurable. 
Now let $z(t)=\dot{x}_{0}(t)-\operatorname{proj}_{Z(t)} \dot{x}_{0}(t)$. For $t \in C$ we have $z(t) \neq 0$. As $z$ is measurable and $m^{*}(C)=1$ so $z(t) \neq 0$ a.e. in $I$. We can thus put $u(t)=z(t) /|z(t)|$. The functions $q$ and $u$ have the required properties.

Proof OF THEOREM 3.3: We modify first the multifunction $F$ along the solution $x_{0}$. For $x=x_{0}(t)$ we put

$$
\hat{F}(t, x)=F(t, x) \cap\left\{y \in \mathbf{R}^{d} ;\langle y, u(t)\rangle \leqslant\left\langle\dot{x}_{0}(t), u(t)\right\rangle\right\}
$$

and $\hat{F}(t, x)=F(t, x)$ if $x \neq x_{0}(t) . \hat{F}$ has exactly the same properties as $F, \dot{x} \epsilon$ $\hat{F}(t, x)$ has the same solutions as (3.1) and $\dot{x}_{0}(t)$ lies on the support hyperplane to $\hat{F}\left(t, x_{0}(t)\right)$ orthogonal to $u(t)$. This allows us to apply Theorem 3.1 for $k=d-1$, that means for one function $e_{1}=u$, and get the convergence of $\left\langle u(\cdot), \dot{x}_{n}(\cdot)-\dot{x}_{0}(\cdot)\right\rangle$ in measure to zero.

Now let us fix $\varepsilon>0$. The function $h$ is measurable and positive valued so there exists $\delta>0$ such that the measure of the set $D_{\delta}=\{t \in I ; h(t) \leqslant \delta\}$ is less than $\varepsilon / 3$. Let

$$
\begin{aligned}
& U_{n}=\left\{t \in I ;\left|<u(t), \dot{x}_{n}(t)-\dot{x}_{0}(t)\right| \geqslant \delta\right\}, \\
& V_{n}=\left\{t \in I ;\left|x_{n}(t)-x_{0}(t)\right| \geqslant q(t)\right\} .
\end{aligned}
$$

We can choose such $k$ that $m\left(U_{n}\right) \leqslant \varepsilon / 3$ and $m\left(V_{n}\right) \leqslant \varepsilon / 3$ for $n \geqslant k$.

Let $n \geqslant k$. Then $m\left(D_{\delta} \cup U_{n} \cup V_{n}\right)<\varepsilon$ and we shall see that for $t \in$ $I \backslash\left(D_{\delta} \cup U_{n} \cup V_{n}\right)$ the equality $x_{n}(t)=x_{0}(t)$ holds. Let us suppose the contrary, that is $x_{n}(t) \neq x_{0}(t)$. As $t \notin V_{n}$ so $\left|x_{n}(t)-x_{0}(t)\right|<q(t)$. Thus $\left\langle u(t), \dot{x}_{0}(t)-\dot{x}_{n}(t)\right\rangle \geqslant h(t)$. $t \notin D$ implies $h(t)>\delta$, hence $\left\langle u(t), \dot{x}_{0}(t)-\dot{x}_{n}(t)\right\rangle>\delta$. On the other hand $t \notin U_{n}$, so $\left|\left\langle u(t), \dot{x}_{0}(t)-\dot{x}_{n}(t)\right\rangle\right|<\delta$. This contradiction proves that the inequality $x_{n}(t) \neq x_{0}(t)$ is not possible and ends the proof.

Here is the most simple example of the situation described in Theorem 3.3.

Example 3.5. We define $F: I \times \mathbf{R} \rightarrow C o(\mathbf{R})$ as follows: $F(t, x)=\{1\}$ if $x \neq 0$ and $[0,1]$ if $x=0$. In fact $F$ does not depend on $t$. The special solution is $x_{0}(t)=0$.

\section{AN APPLiCATION to CONTROL SYSTEMS}

In this section we shall apply Corollary 3.2 to control systems of the form

$$
\dot{x}=f(t, x, u)
$$

where $f: I \times \mathbf{R}^{d} \times \mathbf{R}^{w} \rightarrow \mathbf{R}^{d}, I=[a, b]$. We assume that $f$ satisfies the following conditions:

(a2) $f(\cdot, x, u$,$) measurable for all x, u$; 
(b2) $f(t, \cdot, u)$ Lipschitzian for all $t, u$ with a Lipschitz constant $M(t)$ such that the function $M(\cdot)$ is integrable;

(c2) $f(t, x \cdot)$ continuous for all $t, x$;

(d2) $|f(t, x, u)| \leqslant g_{1}(t)|x|+g_{2}(t)$, where $g_{1}, g_{2}$ integrable.

For each $(t, x)$ the control parameter $u$, which may appear in $f(t, x, u)$, must belong to a set $U(t, x)$; the multifunction $U: I \times \mathbf{R}^{d} \rightarrow \operatorname{Comp}\left(\mathbf{R}^{w}\right)$ will satisfy the conditions:

(e2) for every $\varepsilon>0$ there is a closed set $I_{\varepsilon} \subseteq I$ for which $m\left(I \backslash I_{e}\right)<\varepsilon$ and the restriction of $U$ to $I_{\varepsilon} \times \mathbf{R}^{d}$ is upper semicontinuous with respect to $(t, x)$;

(f2) $|u| \leqslant g_{3}(t)$ for $u \in U(t, x)$, where $g_{3}$ is integrable.

For a fixed measurable function $u: I \rightarrow \mathbf{R}^{\boldsymbol{w}}$ the differential equation

$$
\dot{x}=f(t, x, u(t))
$$

has, for aný initial condition $x(a)$, a unique solution $x: I \rightarrow \mathrm{R}^{d}$. If a.e. in $I$ the condition $u(t) \in U(t, x(t))$ holds then we say that the pair $(x(\cdot), u(\cdot))$ is admissible.

We put

$$
F(t, x)=\{f(t, x, u) u \in U(t, x)\} .
$$

$F$ is a multifunction defined on $I \times \mathrm{R}^{d}$ and with values in $\operatorname{Comp}\left(\mathrm{R}^{d}\right)$. Its properties will be given in Lemma 4.2 .

For $t \in I, x \in \mathbb{R}^{d}, v \in \mathbf{R}^{w}$ we shall denote by $\Phi(t, x, v)$ the set of all $u \in U(t, x)$ such that $f(t, x, u)=v$, that means

$$
\Phi(t, x, v)=(f(t, x, \cdot))^{-1}(v) \cap U(t, x) .
$$

Thus we have defined a multifunction $\Phi: I \times \mathbf{R}^{d} \times \mathbf{R}^{d} \rightarrow \operatorname{Comp}\left(\mathbf{R}^{w}\right)$.

Now we fix an admissible pair $\left(x_{0}, u_{0}\right)$.

THEOREM 4.1. Let us suppose that a.e. in I

$$
\dot{x}_{0}(t) \in \operatorname{Ext}\left(\operatorname{conv}\left(F\left(t, x_{0}(t)\right)\right)\right)
$$

and let $\left(x_{n}, u_{n}\right)$ be a sequence of admissible pairs such that the solutions $x_{n}$ are pointwise convergent to $x_{0}$. Then the sequence of functions

$$
h_{n}(t)=\operatorname{dist}\left(u_{n}(t), \Phi\left(t, x_{0}(t), \dot{x}_{0}(t)\right)\right)
$$

is convergent in measure to zero.

Before proving this theorem we shall give three necessary lemmas. Let us remark that in view of $(\mathrm{d} 2)$ all the values of the functions $x_{n}, x_{0}$ are contained in some closed ball $B \subseteq \mathbf{R}^{d}$. 
LEMMA 4.2. The restriction $F: I \times B \rightarrow \operatorname{Comp}\left(\mathrm{R}^{d}\right)$ is integrably bounded and satisfies the condition (a1).

Proof: The integral boundedness is implied by (d2) and the boundedness of $B$. To prove (a1) let us fix an $\varepsilon>0$ and let $I_{\varepsilon}$ be such a closed subset of $I$ that the following conditions are true:

(a3) $m\left(I \backslash I_{e}\right)<\varepsilon$;

(b3) $\left.f\right|_{I_{c} \times B \times R^{w}}$ is continuous with respect to $(t, x, u)$ (this can be achieved due to (a2)-(d2) and the Theorem of Scorza-Dragoni [10]);

(c3) the set $f\left(I \varepsilon \times B \times \mathrm{R}^{w}\right)$ is precompact in $\mathrm{R}^{d}$;

(d3) the restriction of $U$ to $I_{e} \times \mathbf{R}^{d}$ is upper semicontinuous with respect to $(t, x)$.

The last condition implies that the union of $U(t, x)$, for $(t, x) \in I_{\varepsilon} \times B$, is compact.

By (c3) the graph $G\left(\left.F\right|_{I_{\varepsilon} \times B}\right)$ is bounded in $I \times B \times \mathbf{R}^{d}$. We shall show that it is closed, thus compact. The compactness of the graph implies the upper semicontinuity of a multifunction and this will complete our proof.

So let $\left(t_{n}, x_{n}, v_{n}\right) \rightarrow\left(t_{0}, x_{0}, v_{0}\right)$, where $t_{n} \in I_{\varepsilon}, x_{n} \in B, v_{n} \in F\left(t_{n}, x_{n}\right)$. There exist $u_{n} \in U\left(t_{n}, x_{n}\right)$ such that $v_{n}=f\left(t_{n}, x_{n}, u_{n}\right)$. The sequence $u_{n}$ has a convergent subsequence $u_{n}^{\prime} \rightarrow u_{0} \in U\left(t_{0}, x_{0}\right)$. From (b3) we have. $f\left(t_{0}, x_{0}, u_{0}\right)=v_{0}$ and thus $v_{0} \in F\left(t_{0}, x_{0}\right)$. So the graph $G\left(\left.F\right|_{I_{k} \times B}\right)$ is actually closed.

In the following lemmas $X, Y, Z$ will denote metric spaces.

LEMMA 4.3. Let $\psi: X \times Y \rightarrow Z$ be a continuous function. Then the multifunction $\Psi: X \times Z \rightarrow C l(Y)$ defined by $\Psi(x, z)=(\psi(x, \cdot))^{-1}(z)$ has closed graph.

Its proof is immediate as is the proof of the next lemma.

LEMma 4.4. Let $\Psi: X \rightarrow \operatorname{Comp}(Y)$ be upper semicontinuous, and let the sequence of measurable functions $w_{n}: I \rightarrow X$ converge in measure to a measurable function $w_{0}$. Then the sequence of functions $\mathcal{H}_{n}(t)=e\left(\Psi\left(w_{n}(t)\right), \Psi\left(w_{0}(t)\right)\right)$ converges in measure to zero.

Proof of Theorem 4.1: From Lemma 4.2 we deduce that the multifunction conv $(F(t, x))$ satisfies on $\mathbb{1} \times B$ the conditions (a1), (b1). By (4.3) Corollary 3.2 implies that the sequence $\dot{x}_{n}$ is convergent to $\dot{x}_{0}$ in measure.

We fix $\varepsilon>0$ and let $I_{\varepsilon}$ be a closed subset of $I$ for which conditions (a3)-(d3) are satisfied. From Lemma 4.2 we get the upper semicontinuity of $\left.\Phi\right|_{I_{\epsilon} \times B \times R^{d}}$ with respect to $(t, x, v)$.

The sequence of functions $z_{n}(t)=\left(t, x_{n}(t), \dot{x}_{n}(t)\right)$ converges in measure to the function $z_{0}(t)=\left(t, x_{0}(t), \dot{x}_{0}(t)\right)$. Lemma 4.4 implies then that the $\phi_{n}(t)=$ 
$e\left(\Phi\left(t, x_{n}(t), \dot{x}_{n}(t)\right), \Phi\left(t, x_{0}(t), \dot{x}_{0}(t)\right)\right)$ converge in measure to zero. We have $u_{n}(t) \in$ $\Phi\left(t, x_{n}(t), \dot{x}_{n}(t)\right)$ thus $h_{n}$ converge on $I_{\varepsilon}$ to zero in measure. As $\varepsilon$ was arbitrary, we get the convergence of $h_{n} \rightarrow 0$ in measure on all $I$.

\title{
REFERENCES
}

[1] J.P. Aubin and A. Cellina, Differential inclusions (Springer-Verlag, Berlin, Heidelberg, New York, Tokyo, 1984).

[2] E.J. Balder, 'On weak convergence implying strong convergence in $L_{1}$-spaces', Bull. Austral. Math. Soc. 33 (1986), 363-368.

[3] C. Castaing and M. Valadier, Convex analysis and measurable multifunctions (Springer-Verlag, Berlin, Heidelberg, New York, 1977).

[4] K. Floret, Weakly compact sets (Springer-Verlag, Berlin, Heidelberg, New York, 1988).

[5] J. Jarnik and J. Kurzweil, 'On conditions on right hand sides of differential relations', Casopis Pest. Mat. 102 (1977), 334-349.

[0] C. Olech, 'Extremal solutions of a control system', J. Differential Equations 2 (1966), 74-101.

[7] C. Olech, 'Existence theory in optimal control', in Control theory and topics in functional analysis, I (Vienna, 1976). pp. 291-328.

[8] R.T. Rockafellar, Convex analysis (Princeton University Press, 1970).

[9] T. Rzezuchowski, 'Scorza-Dragoni type theorem for upper semicontinuous multivalued functions', Bull. Acad. Polon. Sci. Sér. Sci. Math. 28 (1980), 61-66.

[10] G. Scorza-Dragoni, 'Un teorema sulle funzioni continue rispetto ad una e misurabili respetto ad un'altra variabile', Rend. Sem. Mat. Univ. Padova 17 (1948), 102-108.

[11] A. Visintin, 'Strong convergence results related to strict convexity', Comm. Partial Differential Equations 9 (1984), 439-466.

\author{
Institute of Mathematics \\ Warsaw Technical University \\ Pl.J.Robotniczej 1 \\ 00-661 Warsaw \\ Poland
}

